\title{
Annual Neck Ultrasonography Surveillance between 3 to 12 Years after Thyroid Lobectomy for Papillary Thyroid Microcarcinoma
}

\author{
Jin Gu Kang, Jung Eun Choi, Soo Jung Lee and Su Hwan Kang \\ Department of Surgery, Yeungnam University College of Medicine, Daegu, Korea
}

\begin{abstract}
Background and Objectives: In South Korea, neck ultrasonography (US) has been used widely at 1- or 2-year intervals to detect recurrence after thyroidectomy. The aim of this study is to assess the prevalence and pattern of recurrence and to establish a proper frequency of follow-up neck US after thyroid lobectomy for papillary thyroid microcarcinoma (PTMC). Materials and Methods: We retrospectively reviewed 262 consecutive patients who had undergone thyroid lobectomy for PTMC from January 2005 to October 2009. They were divided into two groups based on the presence of recurrence (240 patients in group 1 and 22 patients in group 2). The comparison between the two groups involved clinical and pathological characteristics. Results: Recurrences were found in 22 (8.4\%) of 262 patients with 132.5 months follow-up (range, 120-180 months). There was a significant difference in the mean number of follow-up neck US after 5 years postoperatively, shortened interval, total number of follow-up neck US $(p=0.002, p<0.001, p<0.001)$. All recurrences were found between 2-3 to 11-12 years. Recurrences after 5 years were found in $16(72.7 \%)$ of 22 patients. Conclusion: Recurrence after thyroid lobectomy for PTMC occurred with various times during follow up. The minimum frequency of follow-up neck US was not enough for detecting tumor recurrence, especially after 5 years postoperatively. Our results suggest that annual neck US may be appropriate between 3 to 12 years after thyroid lobectomy for PTMC.
\end{abstract}

Key Words: Papillary thyroid microcarcinoma, Recurrence, Ultrasonography

\section{Introduction}

Papillary thyroid microcarcinoma (PTMC) is defined as a $1-\mathrm{cm}$ or smaller tumor, and the detection rate has increased worldwide. It is usually considered to be an indolent disease because it grows slowly and has excellent prognosis after surgical treatment. A recent study reported that there were $8 \%$ tumor enlargement, $3.8 \%$ node metastasis and $6.8 \%$ clinical progression among of 1235 PTMC patients with non-surgical observation. ${ }^{1)}$ Active surveillance can be selectively indicated in older patients. It remains unclear whether active surveillance is better than surgery or not for PTMC. In South Korea, surgical treatment is still widely accepted because there are few complications. The 2015 American Thyroid Association (ATA) guideline recommends that thyroid lobectomy alone is sufficient treatment for PTMC without clinical evidence of aggressiveness. It also reported that PTMC revealed a disease-specific mortality rate of less than $1 \%$ and a recurrence rate of 1 to $2 \%{ }^{2}$ )

Neck ultrasonography (US) is the most useful imaging modality for detecting a recurring lesion after thyroidectomy, but there is no guideline about the frequency and interval of neck US after lobectomy.

Received July 7, 2020 / Revised July 27, 2020 / Accepted July 28, 2020

Correspondence: Su Hwan Kang, MD, PhD, Department of Surgery, Yeungnam University College of Medicine, 170, Hyunchoong-ro, Namgu, Daegu 42415, Korea

Tel: 82-53-620-3580, Fax:82-53-624-1213, E-mail: kangsuhwan@yu.ac.kr

Copyright (c) the Korean Thyroid Association. All rights reserved.

(1) (8) This is an open-access article distributed under the terms of the Creative Commons Attribution Non-Commercial License (http://creative(c) (1) \& 8 commons.org/licenses/by-nc/4.0/), which permits unrestricted non-commercial use, distribution, and reproduction in any medium, provided the original work is properly cited. 
Recurrence after thyroid lobectomy for PTMC was low, and a recurred tumor was not detected in follow-up neck US $\leq 4$ years. Thus, early post-lobectomy neck US in short intervals may be unnecessary. ${ }^{3)} \mathrm{A}$ recent study also reported that the tumor recurrence/persistence rate was $2 \%$; a single session of follow-up neck US is sufficient within 5 years postoperatively. ${ }^{4)}$ Another study reported that a 1- or 2-year neck US interval may be excessive, and only 1 or 2 sessions of follow-up neck US may be sufficient within 10 years postoperatively. ${ }^{5)}$

In South Korea, neck US has been used widely at 1- or 2-year intervals for detecting recurrence after thyroidectomy. The aim of this study is to assess the prevalence and pattern of recurrence and to establish a proper interval of follow-up neck US after thyroid lobectomy for PTMC.

\section{Materials and Methods}

\section{Patients}

We retrospectively reviewed 262 consecutive patients who had undergone thyroid lobectomy for PTMC from January 2005 to October 2009. Thyroid lobectomy was defined as the removal of either the right or the left lobe together with the isthmus. All lobectomies were done with a low collar incision.

The inclusion criteria were:

(1) patients who had undergone thyroid lobectomy regardless of prophylactic central neck dissection;

(2) final pathology report revealed papillary thyroid carcinoma of $1 \mathrm{~cm}$ or less;

(3) follow-up neck US for at least 10 years.

The exclusion criteria were:

(1) follow-up US less than 10 years

(2) missing a certain period during follow up.

There were 582 patients who had undergone lobectomy for PTMC and were initially included in this study. Among them, 320 patients (54.9\%) were transferred to other, smaller hospitals for convenience of examination in various periods postoperatively. Finally, for 262 patients who regularly got follow-up neck US in our hos- pital, we could identify the presence of recurrence. They were divided into two groups based on the presence of recurrence (240 patients in group 1 and $22 \mathrm{pa}-$ tients in group 2). The comparison between the two groups involved clinical (age, gender, frequency, and interval of follow-up neck US) and pathological characteristics (tumor size, location, multifocality, lymphocytic thyroiditis, minimal extrathyroidal extension [ETE], and central lymph-node [LN] metastasis).

\section{Follow-up US and Recurrence}

Neck US was done first at 6 months postoperatively and at 1-year interval thereafter by experienced radiologists using a high-resolution instrument (iU 22; Philips Medical Systems, Bothell, WA, USA) equipped with a 5to $12 \mathrm{MHz}$ linear probe. The interval of follow-up neck US was slightly different for each patient. A shortened interval was defined as changing an interval compared to the previous interval. US findings of suspicious malignancy usually included marked hypoechogenicity, a taller-than-wide shape, ill-defined margin, and internal microcalcifications. Recurrence was defined as the presence of a new papillary cancer proven by fine-needle aspiration cytology (FNAC) among the operative bed, residual lobe, or central or lateral neck LN. A multidisciplinary team (endocrine surgeons and radiologists) assessed every follow-up neck US finding. Indeterminate lesion on operative bed was defined as US finding closer to postoperative change or granuloma. Indeterminate LN was defined as loss of central hilar echo and absence of central hilar vascularity. And indeterminate nodule was defined as TR3 by Thyroid Imaging Reporting and Data System (TI-RADS). Convenience with other examinations means that neck US was performed when patients visited to the hospital for other department examination like as breast US, brain MRI (magnetic resonance imaging), chest $X$-ray and laboratory test. If there was a suspicious lesion by neck US, FNAC was done in order to rule out recurrence. In recurrence proven by FNAC, the timing of recurrence was defined as the time when the small tumor was initially identified by neck US, not when the pathology result came out. We also analyzed site of recurrence, type of second operation, interval between detection and first 
Annual Neck Ultrasonography Surveillance after Lobectomy for PTMC

operation, interval between second operation and detection, and shortened neck US interval.

\section{Statistical Analyses}

A chi-squared test or Fisher's exact test was used to compare the categorical variables. An independent t-test was used for continuous valuables, which are expressed as mean \pm standard deviation. All statistical analyses were done using IBM SPSS version 21.0 (IBM Co., Armonk, NY, USA) and $p<0.05$ indicated statistical significance.

\section{Results}

\section{Patient Characteristics}

All 262 patients who had undergone lobectomy were diagnosed as PTMC based on the final pathologic result. The mean age was $45.0 \pm 8.9$ and $43.8 \pm 8.1$ years. Among the 262 patients, $227(94.6 \%)$ and 21 (95.5\%) were female in the two groups. The dominant lobe of PTMC included the right $(n=145)$, the left $(n=116)$, and the isthmus $(n=1)$. Tumor location was upper $(n=39)$, middle $(n=180)$, or lower $(n=43)$. The mean of tumor size was $5.8 \pm 2.4$ and $5.9 \pm 2.4 \mathrm{~mm}$. Contralateral nodule was detected in $15(6.3 \%)$ and 1 (4.5\%). Multifocality was found in $38(15.8 \%)$ and 7 $(31.8 \%)$, and lymphocytic thyroiditis was found in 51 (21.3\%) and 1 (4.5\%) in groups 1 and 2 respectively. Central neck dissection (CND) was performed in 173 (66\%) patients who were identified as having enlarged LNs intraoperatively. According to the American Joint Committee on Cancer (AJCC) $8^{\text {th }}$ edition, all patients were T1a and 11 patients (4.2\%) were N1a. All ETE

Table 1. Clinicopathological characteristics of patients

\begin{tabular}{|c|c|c|c|}
\hline Characteristic & Group $1(n=240)$ & Group $2(n=22)$ & $p$ value \\
\hline Age (yr) & $45.0 \pm 8.9$ & $43.8 \pm 8.1$ & 0.520 \\
\hline Gender & & & 0.086 \\
\hline Female & $227(94.6)$ & $21(95.5)$ & \\
\hline Male & $13(5.4)$ & $1(4.5)$ & \\
\hline Dominant lobe & & & 0.401 \\
\hline Right & $131(54.6)$ & $14(63.6)$ & \\
\hline Left & $108(45)$ & $8(36.4)$ & \\
\hline Isthmus & $1(0.4)$ & 0 & \\
\hline Tumor location & & & 0.339 \\
\hline Upper & $32(13.3)$ & $7(31.8)$ & \\
\hline Middle & $170(70.8)$ & $10(45.5)$ & \\
\hline Lower & $38(15.8)$ & $5(22.7)$ & \\
\hline Multifocality & $38(15.8)$ & $7(31.8)$ & 0.074 \\
\hline Contralateral nodule & $15(6.3)$ & $1(4.5)$ & NA \\
\hline Lymphocytic thyroiditis & $51(21.3)$ & $1(4.5)$ & 0.089 \\
\hline Tumor size $(\mathrm{mm})$ & $5.8 \pm 2.4$ & $5.9 \pm 2.4$ & 0.774 \\
\hline T1 a* & $240(100)$ & $22(100)$ & NA \\
\hline $\mathrm{N} \mathrm{a}^{\dagger}$ & $10(4.2)$ & $1(4.5)$ & NA \\
\hline CND & $158(65.8)$ & $15(68.2)$ & 0.824 \\
\hline Minimal ETE & $124(51.7)$ & $16(72.7)$ & 0.058 \\
\hline Number of performed US ( $\leq 5 \mathrm{yr}$ ) & $4.7 \pm 0.8$ & $5.1 \pm 1.2$ & 0.065 \\
\hline Number of performed US ( $>5 \mathrm{yr}$ ) & $5.6 \pm 1.3$ & $7.0 \pm 1.9$ & 0.002 \\
\hline Shortened US interval & $37(15.4)$ & $15(68.2)$ & $<0.001$ \\
\hline Total number of performed US & $10.3 \pm 1.5$ & $12.1 \pm 2.1$ & $<0.001$ \\
\hline $\begin{array}{l}\text { Interval between last US and } \\
\text { 1st operation (month) }\end{array}$ & $132.0 \pm 12.2(121-180)$ & $138.0 \pm 10.6(120-159)$ & 0.027 \\
\hline
\end{tabular}

*T1a tumor $\leq 1 \mathrm{~cm}$ in greatest dimension.

${ }^{\dagger} \mathrm{N} 1$ a metastasis to level VI or VII lymph nodes.

Values are presents as mean \pm standard deviation or number (\%).

CND: central neck dissection, ETE: extrathyroidal extension, LN: lymph node, NA: not applicable, US: ultrasonography 
was minimal and was found for 124 (51.7\%) and 16 $(72.7 \%)$ in the two groups. The mean number of follow-up neck US during the first 5 years was $4.7 \pm 0.8$ and $5.1 \pm 1.2(p=0.065)$, but the mean number of follow-up neck US after 5 years postoperatively was $5.6 \pm 1.3$ and $7.0 \pm 1.9$ respectively ( $p=0.002)$. Shortened neck US interval was $37(15.4 \%)$ and $15(68.2 \%)(p<$ 0.001 ); total number of follow-up neck US was $10.3 \pm 1.5$ and $12.1 \pm 2.1(p<0.001)$; and interval between first operation and last neck US was $132.0 \pm$ 12.2 and $138.0 \pm 10.6$ months $(p=0.027)$ in groups 1 and 2 respectively. There was a significant difference in the mean number of follow-up neck US after 5 years postoperatively, shortened interval, total number of follow-up neck US, and interval between the first operation and last neck US (Table 1).

\section{Shortened Neck US Interval}

The factors resulting in shortened neck US interval included indeterminate lesion on the operative bed, indeterminate central and lateral LN, preexisting con- tralateral nodule, new contralateral nodule, and convenience with other examinations. Indeterminate lesion on the operative bed was $18(7.5 \%)$ and $2(9.1 \%)$. Preexisting contralateral nodule was $5(2.1 \%)$, and convenience with another examination was $15(6.3 \%)$ only in group 1. Indeterminate central LN was $9(3.8 \%)$ and $4(18.2 \%)(p=0.016)$, indeterminate lateral LN was $4(1.7 \%)$ and $4(18.2 \%) \quad(p=0.002)$; and new contralateral nodule was $92(38.3 \%)$ and $16(72.7 \%)$ $(p=0.002)$ in groups 1 and 2, respectively. There were 2 indeterminate operative bed; there were 4 indeterminate central LNs (3 metastases), 5 indeterminate lateral LNs (5 metastases) and 16 new contralateral nodules (14 metastases) with some overlap. There was a significant difference in indeterminate central and lateral LN and new contralateral nodules between the two groups (Table 2).

\section{Recurrence after Thyroid Lobectomy}

Recurrences were found in $22(8.4 \%)$ of 262 patients with 132.5 months follow-up (range, 120-180 months).

Table 2. Factors affect to shortened neck US interval during follow up

\begin{tabular}{lccc}
\multicolumn{1}{c}{ Factor } & Group $1(n=240)$ & Group 2 $(n=22)$ & $p$ value \\
\hline Indeterminate lesion on the bed & $18(7.5)$ & $2(9.1)$ & 0.679 \\
Indeterminate central LN* & $9(3.8)$ & $4(18.2)$ & 0.016 \\
Indeterminate lateral LN* & $4(1.7)$ & $5(22.7)$ & 0.002 \\
Preexisting contralateral nodule & $5(2.1)$ & $0(0)$ & NA \\
Newly contralateral nodule* & $92(38.3)$ & $16(72.7)$ & 0.002 \\
Convenience with other examination & $15(6.3)$ & $0(0)$ & 0.623 \\
\hline
\end{tabular}

*Values are overlapped.

Values are presents as number (\%).

LN: Iymph node, NA: not applicable, US: ultrasonography

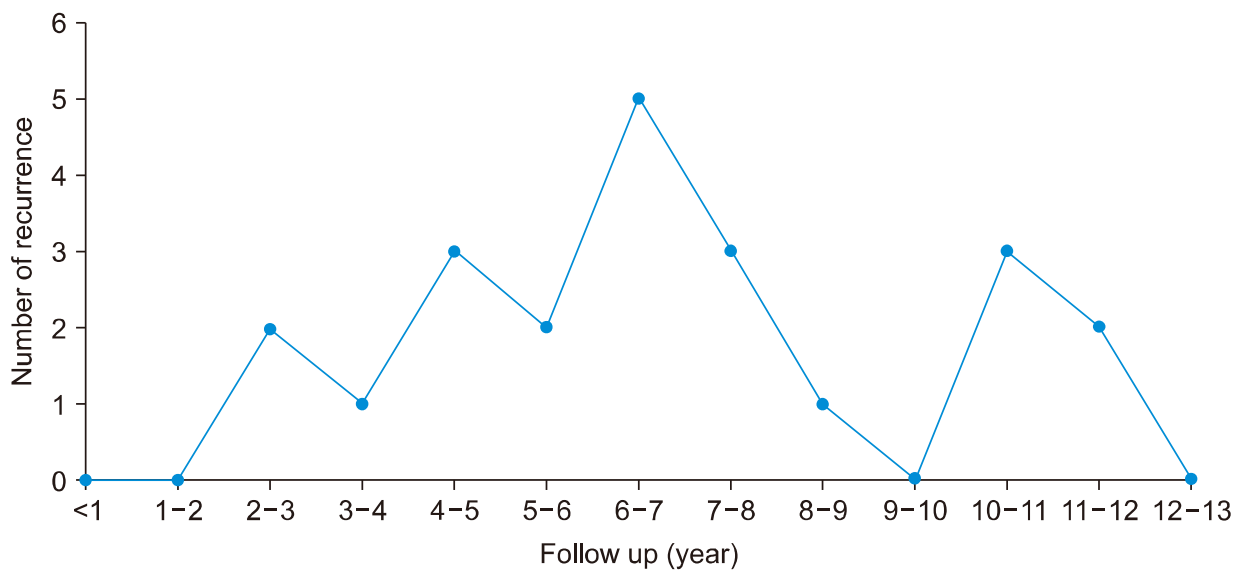

Fig. 1. Frequency of recurrence during follow up. 
Annual Neck Ultrasonography Surveillance after Lobectomy for PTMC

Recurrence revealed at various times during follow up. Recurrences were found in 2 patients (9.1\%) for 2-3 years, 1 patient $(4.5 \%)$ for $3-4$ years, 3 patients (13.6\%) for 4-5 years. Recurrence after 5 years were found in 16 patients (72.7\%) (Fig. 1). The site of recurrence involved $14(63.6 \%)$ residual lobes, 3 (13.6\%) central LNs, and 5 (22.8\%) lateral LNs. The mean of recurred tumor size was $2.4 \pm 2.2 \mathrm{~mm}$, and the interval between detection and the first operation was $80.7 \pm$ 31.9 months. The second operation included 5 (23.8\%) completion thyroidectomy, 11 (52.4\%) completion thyroidectomy with CND, and 5 (23.8\%) completion thyroidectomy with ipsilateral modified radical neck dissection (MRND). There was one case of active surveillance, because the patient was 65 years and wanted observation without surgery. In the observation case, the first operation was a right lobectomy with CND ( $p$ T1N0), and recurrence was identified on the left lobe with a 4-mm tumor after 103 months postoperatively. Tumor size was slightly increased by $2 \mathrm{~mm}$ for 35 months after recurrence. More than 6 -month intervals between the second operation and detection was found in 5 (23.8\%) patients $(6,7,18$, 46, 61 months). Increased tumor size after detection was found in 4 of 5 patients $(1.4 \mathrm{~mm}$ over 7 months, $3 \mathrm{~mm}$ over 18 months, $3.2 \mathrm{~mm}$ over 46 months, and $1.5 \mathrm{~mm}$ over 61 months) (Table 3).

\section{Discussion}

PTMC is defined as a tumor $1.0 \mathrm{~cm}$ or smaller in diameter and generally has a good prognosis. ${ }^{6)}$ The prevalence of PTMC has recently increased related to the progress in both neck US and FNAC. ${ }^{7,8)}$ According to the 2015 ATA guidelines, thyroid lobectomy is sufficient for a tumor $<1 \mathrm{~cm}$ without ETE or cNO carcinomas. The initial surgery of thyroid carcinoma $>1$ $\mathrm{cm}$ and $<4 \mathrm{~cm}$ without ETE, cNO can be near-total or total thyroidectomy or lobectomy. ${ }^{2}$ For patients who had undergone thyroid lobectomy for PTMC without other adverse factors, long-term follow-up was not recommended. ${ }^{9)}$ After thyroid lobectomy with low-risk PTC, minimal ETE is an important prognostic factor for recurrence. ${ }^{10)}$ Tumor size is known to be one of the
Table 3. Details of recurrence $(n=22)$

\begin{tabular}{lr}
\multicolumn{1}{c}{ Characteristic } & $\begin{array}{c}\text { Number of } \\
\text { cases }\end{array}$ \\
\hline Site of recurrence & \\
Residual lobe & $14(63.6)$ \\
Central LN & $3(13.6)$ \\
Lateral LN & $5(22.8)$ \\
Tumor size (mm) & $2.4 \pm 2.2$ \\
Interval between detection and 1st & $80.7 \pm 31.9$ \\
operation (month) & \\
2nd operation & $21(95.5)$ \\
$\quad$ Completion thyroidectomy & $5(23.8)$ \\
Completion thyroidectomy with CND & $11(52.4)$ \\
Completion thyroidectomy with & $5(23.8)$ \\
$\quad$ ipsilateral MRND & \\
Interval between 2nd operation and & $21(95.5)$ \\
detection (month interval) & $16(76.2)$ \\
$\quad<6$ & $5(23.8)$ \\
$\quad 66$ & $1(4.5)$ \\
Observation & $4(19)$ \\
Increased tumor size after detection &
\end{tabular}

Values are presents as mean \pm standard deviation or number (\%).

CND: central neck dissection, LN: lymph node, MRND: modified radical neck dissection

most important prognostic factors of PTMC, but it is controversial. Some authors reported that a tumor size larger than $5 \mathrm{~mm}$ is a risk factor for decreasing survival and recurrence in PTMC. ${ }^{11)}$ Other studies showed that a PTMC larger than $8 \mathrm{~mm}$ was more aggressive. ${ }^{12)}$ Multivariate analysis showed that there was no correlation between tumor size and LN metastasis or recurrence. Postoperative hypothyroidism was also not a risk factor for recurrence after lobectomy with PTC. ${ }^{13,14)}$ LN metastasis was considered to be a more important prognostic factor than was tumor size. ${ }^{15)}$ Other multivariate analysis showed that coexisting benign nodules of the contralateral lobe, multifocality, and tumor size larger than $5 \mathrm{~mm}$ were independent predictive factors for contralateral occult carcinoma. ${ }^{16)}$ Recurrence after thyroid lobectomy for PTMC has been reported to be $8 \% .{ }^{15)}$ Other studies reported that the recurrence rate was only $1.5 \%$, but this was related to the short follow-up of less than 90 months. ${ }^{3)}$ The recurrence rate after lobectomy for PTC was $6.5 \%$ in the residual lobe, $9.4 \%$ in the regional LNs, and $6.4 \%$ in the distant organs at 25 years after the first 
operation. ${ }^{17)}$ The other study reported that the recurrence rate was $2.6 \%$ with the average 48.7 months follow-up. ${ }^{18)}$ In our study, 22 (8.4\%) of 262 patients revealed recurrence and this rate is higher than other studies. 582 patients had undergone thyroid lobectomy in our hospital and 320 of 582 were transferred to other, smaller hospitals after the first operation. If all patients who had undergone thyroid lobectomy were involved, the recurrence rate could be 3.8\% (22 of 582 patients) and was similarly low compared to other studies. But it is also hard to rule out the possibility of actual increased recurrence rate over time. To assess an accurate prevalence and pattern of recurrence over time, we enrolled only 262 patients who had regular follow-up neck US for more than 10 years postoperatively in our hospital.

After thyroidectomy, there are a few modalities for surveillance. Serum thyroglobulin $(\mathrm{Tg})$ levels have a less important role in surveillance after lobectomy. An increasing trend of $\mathrm{Tg}$ levels was found to be about $10 \%$ annually, and clinical implications of serum $\mathrm{Tg}$ level after lobectomy are controversial. Periodic Tg level was not a useful predictive factor for recurrence. ${ }^{19)}$ After surgery for PTC, neck US is commonly used to detect recurrence, and it provides the tumor size, echogenicity, shape, and vascularity. ${ }^{20,21)}$ There are no published guidelines about follow-up US after thyroid lobectomy for PTMC. In a multicenter study, the tumor recurrence rate was low $(2.0 \%)$ after thyroid lobectomy for PTMC, and recurrence was detected with a mean interval of $42.9 \pm 25.9$ months. Thus, 1 - or 2-year intervals may be unnecessary, and one followup US is sufficient within the first 5 years postoperatively. ${ }^{4)}$ Other studies showed that tumor recurrence was $2.8 \%$, and $82(45.8 \%)$ of the 179 patients had more frequency of neck US than 5.2. One or two follow-up USs may be sufficient within the first 10 years after lobectomy for PTMC. ${ }^{5}$ In an observational trial, the rate of increased tumor size was $6.3 \%$ at 5 years and $7.3 \%$ at 10 years of follow-up. Younger patients tended to progress more than older patient did $(9.7 \%$ in the under-50 group vs $6.4 \%$ in over-50 group) at 10 years follow-up. ${ }^{22)}$ The incidence of tumor-size enlargements was $4.9 \%$ at 5 years and $8.0 \%$ at 10 years follow-up. Based on multivariate analysis, being younger was an independent factor for progression of PTMC. 1) Another active surveillance study revealed that the average tumor size at 3 years and 4 years follow-up were significantly larger than the initial size. ${ }^{18)}$ One study reported that there was a higher incidence of LN metastasis in PTMC of more than $5 \mathrm{~mm}$ and a larger tumor of PTMC is related to LN metastasis and advanced stage. ${ }^{23)}$ In our study, there were five cases of delayed second operation and one observation case. Of the 5 delayed second operation cases, 4 showed recurred tumor enlargement $(1.4 \mathrm{~mm}$ over 7 months, $3 \mathrm{~mm}$ over 18 months, $3.2 \mathrm{~mm}$ over 46 months and $1.5 \mathrm{~mm}$ over 61 months). One observation case was an older (65 years) female with a slightly increased tumor size (2 mm for 35 months). Fig. 1 shows frequency of recurrence with various time. It means that it is hard to predict the recurrence time. Recurrences less than 5 years were found in $6(27.3 \%)$ of 22 patients. Eleven of 22 patients had recurrence at 5 to 10 years postoperatively. All recurrences were found between 3 and 12 years after the first operation. Although recurred tumor size was small $(2.4 \pm 2.2 \mathrm{~mm})$ in our study, five lateral neck $L N$ metastases received MRND. These results showed that recurrence might not be always simple, unlike recurrence reported in other studies. ${ }^{4,5)}$ According to an active surveillance trial, the incidence of tumor size enlargement is $4.9 \%$ at 5 years and $8.0 \%$ at 10 years of follow-up. ${ }^{1)}$ Intervals of more than 6 months between the second operation and detection were found in five patients. Four of 21 patients showed increases of tumor size after detection of recurrence in our study. These results may imply that delayed detection of recurrence can be harmful in some patients, although the risk of PTMC is low. In South Korea, it is difficult to follow up for more than 10 years in tertiary hospitals because of complicated procedures. A lot of patients who received thyroid lobectomy for PTMC were transferred to other and smaller hospitals for convenience. Our study involved 262 patients with follow-up of more than 10 years in a single tertiary hospital with consistency. Our study showed recurrence rate of $8.4 \%$ and MRND rate of $1.9 \%$. Although the number of pa- 
Annual Neck Ultrasonography Surveillance after Lobectomy for PTMC

tients included in this study is small, we have to reconsider that minimum frequency of follow-up neck US is appropriate. Our study has some limitations. First, there was no comparison directly with other intervals. Further study is needed to confirm minute neck US interval. Second, the average follow-up duration was 132.5 months (range, 120-180 months) with very little follow-up cases after 12 years. Long-term follow-up over 20 years will be needed to confirm the pattern and rate of recurrence in the future. A multicenter study might also be needed to determine accurate follow-up neck US intervals after thyroid lobectomy for PTMC.

In conclusion, recurrence after thyroid lobectomy for PTMC occurred with various time during follow-up. All recurrences were found between 2-3 to 11-12 years. Recurrences after 5 years were found in 16 (72.7\%) of 22 patients. The minimum frequency of follow-up neck US was not enough for detecting tumor recurrence, especially after 5 years postoperatively. Our results suggest that annual neck US may be appropriate between 3 to 12 years after thyroid lobectomy for PTMC.

\section{Acknowledgments}

Not applicable.

\section{Conflicts of Interest}

No potential conflicts of interest relevant to this article was reported

\section{Orcid}

Jin Gu Kang: https://orcid.org/0000-0002-3154-0697 Jung Eun Choi: https://orcid.org/0000-0003-2290-6228 Soo Jung Lee: https://orcid.org/0000-0003-1202-3974 Su Hwan Kang: https://orcid.org/0000-0002-6508-006X

\section{References}

1) Ito Y, Miyauchi A, Kihara M, Higashiyama T, Kobayashi K, Miya A. Patient age is significantly related to the progression of papillary microcarcinoma of the thyroid under observation. Thyroid 2014;24(1):27-34.

2) Haugen BR, Alexander EK, Bible KC, Doherty GM, Mandel SJ, Nikiforov YE, et al. 2015 American Thyroid Association management guidelines for adult patients with thyroid nodules and differentiated thyroid cancer: the American Thyroid Association guidelines task force on thyroid nodules and differentiated thyroid cancer. Thyroid 2016;26(1):1-133.

3) Kim DW. Long-term follow-up ultrasonography after lobectomy in papillary thyroid microcarcinoma patients: a single-center study. Endocr Res 2016;41(3):213-7.

4) Baek HJ, Kim DW, Lee CY, Huh JY, Sung JY, Choi YJ. Analysis of postoperative ultrasonography surveillance after hemithyroidectomy in patients with papillary thyroid microcarcinoma: a multicenter study. Endocr Pract 2017;23(7):794-802.

5) Choi YS, Kim DW, Lee YJ, Ha TK, Jung SJ, Baek HJ Appropriate neck ultrasonography surveillance during the first 10 years after hemithyroidectomy in papillary thyroid microcarcinoma patients: a single-center study. Ultrasound Q 2019;35(3):275-80.

6) Piersanti M, Ezzat S, Asa SL. Controversies in papillary microcarcinoma of the thyroid. Endocr Pathol 2003;14(3):183-91.

7) Davies L, Welch HG. Increasing incidence of thyroid cancer in the United States, 1973-2002. JAMA 2006;295(18):2164-7.

8) Leenhardt L, Grosclaude P, Cherie-Challine L, Thyroid Cancer Committee. Increased incidence of thyroid carcinoma in France: a true epidemic or thyroid nodule management effects? Report from the French Thyroid Cancer Committee. Thyroid 2004;14(12):1056-60.

9) Perros P, Boelaert K, Colley S, Evans C, Evans RM, Gerrard $\mathrm{Ba} G$, et al. Guidelines for the management of thyroid cancer. Clin Endocrinol (Oxf) 2014;81 Suppl 1:1-122.

10) Park YM, Lee DY, Oh KH, Cho JG, Baek SK, Kwon SY, et al. Clinical implications of pathologic factors after thyroid lobectomy in patients with papillary thyroid carcinoma. Oral Oncol 2017;75:1-5.

11) Lo CY, Chan WF, Lang BH, Lam KY, Wan KY. Papillary microcarcinoma: is there any difference between clinically overt and occult tumors? World J Surg 2006;30(5):759-66.

12) Roti E, Rossi R, Trasforini G, Bertelli F, Ambrosio MR, Busutti L, et al. Clinical and histological characteristics of papillary thyroid microcarcinoma: results of a retrospective study in 243 patients. J Clin Endocrinol Metab 2006;91(6):2171-8.

13) Ahn D, Lee GJ, Sohn JH, Jeon JH. Oncological impact of hypothyroidism and levothyroxine supplementation following hemithyroidectomy in patients with papillary thyroid carcinoma. Head Neck 2020;42(5):1004-13.

14) Lee MC, Kim MJ, Choi HS, Cho SW, Lee GH, Park YJ, et al. Postoperative thyroid-stimulating hormone levels did not affect recurrence after thyroid lobectomy in patients with papillary thyroid cancer. Endocrinol Metab (Seoul) 2019;34(2):150-7.

15) Pisanu A, Reccia I, Nardello O, Uccheddu A. Risk factors for nodal metastasis and recurrence among patients with papillary thyroid microcarcinoma: differences in clinical relevance between nonincidental and incidental tumors. World J Surg 2009;33(3): 460-8.

16) Huang H, Liu S, Xu Z, Ni S, Zhang Z, Wang X. Long-term outcome of thyroid lobectomy for unilateral multifocal papillary 
carcinoma. Medicine (Baltimore) 2017;96(27):e7461.

17) Matsuzu K, Sugino K, Masudo K, Nagahama M, Kitagawa $\mathrm{W}$, Shibuya $\mathrm{H}$, et al. Thyroid lobectomy for papillary thyroid cancer: long-term follow-up study of 1,088 cases. World J Surg 2014;38(1):68-79.

18) Ito $Y$, Uruno T, Nakano K, Takamura $Y$, Miya A, Kobayashi $\mathrm{K}$, et al. An observation trial without surgical treatment in patients with papillary microcarcinoma of the thyroid. Thyroid 2003;13(4):381-7.

19) Park S, Jeon MJ, Oh HS, Lee YM, Sung TY, Han M, et al. Changes in serum thyroglobulin levels after lobectomy in patients with low-risk papillary thyroid cancer. Thyroid 2018; 28(8):997-1003.

20) Frates MC, Benson CB, Charboneau JW, Cibas ES, Clark $\mathrm{OH}$, Coleman BG, et al. Management of thyroid nodules detected at US: society of radiologists in ultrasound consensus conference statement. Radiology 2005;237(3):794-800.

21) Park HK, Kim DW, Ha TK, Heo YJ, Baek JW, Lee YJ, et al. Utility of routine ultrasonography follow-up after total thyroidectomy in patients with papillary thyroid carcinoma: a single-center study. BMC Med Imaging 2018;18(1):12.

22) Fukuoka O, Sugitani I, Ebina A, Toda K, Kawabata K, Yamada K. Natural history of asymptomatic papillary thyroid microcarcinoma: time-dependent changes in calcification and vascularity during active surveillance. World J Surg 2016;40(3): 529-37.

23) Xia S, Dong Y, Kang H, Zhan W. Ultrasonography is valuable in evaluation of papillary thyroid microcarcinoma based on 5 mm tumor size. J Cancer Res Ther 2018;14(9):319-23. 